\title{
Retrieval in cued recall
}

\author{
JOHN L. SANTA \\ Rutgers University, Douglass College, New Brunswick, New Jersey 08903
}

ALAN B. RUSKIN

University of California, Irvine, California 92664

DAVID SNUTTJER

Purdue University, West Lafayette, Indiana 47907

and

\author{
LINDA BAKER \\ Rutgers University, Douglass College, New Brunswick, New Jersey 08903
}

\begin{abstract}
Three experiments examined retrieval in a cued recall paradigm by manipulating the pattern of output cues. In the first two experiments, subjects were paced through recall of a categorized list by recalling one word at a time in response to a category name as a retrieval cue. The pattern of these output cues exerted a strong influence on performance. Subjects recalled considerably more when the cues were blocked by category than when required to recall successive words from different categories. This output effect increased over trials, and was interpreted in terms of a model in which subjects develop a retrieval strategy that can be interfered with by the circumstances of output. The last experiment contrasted structural and strategy models in a hierarchical recall paradigm. Subjects were given a set of postinput recall cues that either made evident the hierarchical structure of the input list or contained the same cue words but with no hierarchical information. Information about the hierarchical structure improved performance even when provided only during recall. The data were discussed in terms of recent models of recall, and a compromise was suggested.
\end{abstract}

For some time now, psychologists have given lip service to the importance of retrieval in the recall paradigm. It is common to distinguish memories which are available but not accessible, and material which is on the tip of the tongue, but cannot be brought to mind; frequent reference is also made to the retrieval stage of recall. It is interesting, therefore, that the notion of a retrieval strategy has received so little empirical documentation. The present paper examines the importance of a retrieval strategy by manipulating the way in which cues are provided during recall. The intent is not to contrast the presence and absence of cues, but rather to ask if the form of cueing influences memory, i.e., are the test circumstances important, and is it possible to affect performance simply by directing this order of recall? Thus, the experiments are concerned with the structure of recall and the importance of a systematic and orderly output.

Such interest in the conditions of output as a means of examining retrieval is motivated by two considerations. First, it is possible to denote a retrieval effect only if a manipulation is effective independent of

Requests for reprints should be sent to John L. Santa, Department of Psychology, Rutgers University, Douglass College, New Brunswick, New Jersey 08903.

The research was in part supported by Biomedical and Research Council grants to the first author. The last experiment was conducted while the first author was on an NIMH postdoctoral fellowship at Stanford University. input factors. Second, there are recent data which uniquely implicate the test trial in the development of a retrieval strategy. Hogan and Kintsch (1971, Experiment II) examined the effects of study and test trials on both recall and recognition tasks. One group of subjects was given four study trials to learn a list of words, and was tested $48 \mathrm{~h}$ later. Another group, which received one study and three test trials, was able to recall more, but recognized less, than the group receiving four study trials. These data support the contention that subjects learn to retrieve while trying to recall.

Given the importance of test trials and the potential influence of output pattern, the next problem is to explain such effects in terms of a general model of memory. Therefore, the present experiments are also concerned with the role of retrieval in contemporary models of recall. To this end, it is useful to distinguish several current approaches to human memory and to outline their treatment of the retrieval concept. One approach emphasizes structural aspects of memory and minimizes the importance of a retrieval strategy. Anderson's (1972) model of recall (FRAN) exemplifies such a structural position. The model, as implemented, attributes recall and organization to the use of a pre-existing associative network and to a study process which "tags" items and the associative connections between them. The recovery of items identified as list members is accomplished through FRAN's retrieval 
algorithm, which searches the associative network by following tagged associations. The retrieval process in this version of FRAN is somewhat subservient in that it depends on the existing associative structure as well as on the process of marking the network during study. The concept of a retrieval strategy in FRAN is perhaps best identified with the selection of key words or entry points to the marked associative structure. FRAN selects a limited number of key words as retrieval cues to access the associative net. Aside from this selection of entry points, the model places a fundamental emphasis on the use of a pre-existing associative network and, for this reason, may be considered a structural model of recall.

In contrast to such structural models are those which emphasize the importance of the retrieval process. Retrieval models can be subdivided into two classes: (a) independent trace models such as those suggested by Slamecka (1968) and Shiffrin (1970), and (b) unitization models similar to that proposed by Tulving $(1968,1972)$. The independent trace models conceive of memory as an unorganized collection of items upon which a retrieval process operates to produce organization and improve recall. They exemplify a pure process conception of memory, stressing the importance of an intelligent processor capable of defining appropriate search sets, and minimizing the role of a structured data base.

In contrast, unitization models (e.g., Tulving, $1968,1972)$ combine both structural information and a strategy emphasis. These models propose that subjects adopt a retrieval strategy and encode information, together with retrieval cues, into a temporary (episodic) memory. Learning in the recall paradigm is viewed as a problem of developing a strategy that unitizes material and improves retrieval. Such models are not structural like Anderson's FRAN, but emphasize the flexibility of encoding with strategies and are not bound by the associative relationships of a semantic memory. On the other hand, neither are they strictly process models since they suggest that subjects rely on semantic memory information to establish a temporary memory structure.

In the present paper, these models of recall are contrasted on their ability to explain the effect of output structure. The first two experiments employ categorized lists, and manipulate the retrieval order by cueing recall of each item with the category name. Cueing each item allows us to direct output in a manner either consistent or inconsistent with a good retrieval strategy. A good output order is quite possibly one where a subject is cued to recall all words from a particular category before proceeding to the next, as opposed to one which requires recall of successive words from different categories. Blocked output should be relatively good because it allows subjects to abstract retrieval cues and to establish units of retrieval among semantically related words.

\section{EXPERIMENT I}

\section{Method}

Materials and Design. Sixty words were selected for the recall list from the Battig and Montague (1968) norms. The list contained 15 items from each of four conceptual categories (trees, animals, countries, and professions). The instances of these categories were all single word nouns whose total frequency of occurrence was less than 11 . The words were typed on plastic and presented with a Kodak Carousel slide projector.

The 60-word list, presented for five study-test trials, was either blocked by category or distributed across categories. For the blocked presentation condition, all 15 words from each of the four categories were presented successively. For the distributed condition, one instance from each category was presented in a specific order, and the procedure was repeated 15 times. A different ordering of the four categories was used on each trial for both presentation conditions.

The two types of input (blocked and distributed) were factorially combined with three types of output, blocked, distributed and free. The subjects were paced through recall one word at a time by being read a category name every $7 \mathrm{sec}$ (blocked and distributed) or by being instructed to recall a word every $7 \mathrm{sec}$ (free). For blocked output, subjects recalled all the words from a category before proceeding to recall from the next category. For distributed output, each category name occurred once, in a particular order, e.g., tree, animal, country, profession, and the order was repeated 15 times. The sequence of cues remained the same across trials, but the initial category cue changed. There were no category constraints on the free output group; subjects were allowed to recall one word from any category during each 7 sec period.

Thus, the experiment consisted of a 2 by 3 by 5 factorial design with two levels of input (blocked and distributed), three levels of output (blocked, distributed, and free), and five trials. A total of 231 introductory psychology students served as subjects. They were tested in groups of 15 to 25 , with two groups of subjects assigned to each of the six conditions.

Procedure. The study list was presented on slides, each slide remaining on the screen for $.8 \mathrm{sec}$, with an ISI of $1.6 \mathrm{sec}$. After presentation of the study list, subjects were given a $45-\mathrm{sec}$ interference task to eliminate any recency effects. The interference task consisted of written free association to high association value nonsense syllables. Immediately following the interference task, subjects began their paced recall which was paced over a tape recorder at a rate of one cue period every $7 \mathrm{sec}$. In the blocked and distributed output conditions, subjects were told a category name and a line number on which to write their response. The subjects in the free output condition were given only the line number. The four category names were written on the blackboard to ensure that subjects in the free output condition would not forget categories from the study list.

\section{Results}

The mean number or words recalled over trials as a function on input and output condition is shown in Figure 1. An unweighted means analysis of variance of these data indicates that the main effects of input, $\mathrm{F}(1,225)=33.3, \quad \mathrm{p}<.001, \quad$ output, $\quad \mathrm{F}(2,225)=4.2$, $\mathrm{p}<.05$ and trials, $F(4,900)=1835.5, p<.001$ were all significant. The Input by Trials interaction was significant $F(4,900)<4.2, p<.01$, as was the Output by Trials interaction $F(8,900)=7.2, p<.001$. However, it should be noted that the interaction of Input by Trials arises primarily from the first trial. The difference between blocked and distributed input is relatively 
constant beyond the first trial, and an analysis of variance discarding this trial leads to a nonsignificant interaction of Input by Trials, $F(3,675)=1.25, p=.29$. The interaction of Output by Trials remains virtually unchanged by discarding the first trial, $F(6,675)=7.60$, $p<.001$. The three-factor interaction of Input by Output by Trials was not significant, $\mathrm{F}<1.00$.

Inspection of Figure 1 shows that blocked input consistently led to better recall than distributed input. The effect of the output conditions was the same for either type of input as reflected in the nonsigificant interaction of Input by Output, $F<1.00$. The type of output has virtually no effect on Trial 1 , but the influence of output grows over trials so that by Trial 5 type of output has as much of an effect as type of input. Figure 1 shows clearly that beyond the first trial recall in the output conditions is in the decreasing order blocked, free, distributed. The contrasts of blocked output vs. free output, $F(1,900)=37.00, p<.001$, and free output vs. distributed output, $F(1,900)=17.32, p<.001$, indicate that all the differences between the output conditions are significant on Trial 5.

\section{Discussion}

The results of Experiment I indicate that manipulations of both input and output pattern have strong effects on recall. The influence of blocked input is by now relatively well documented (Bower, Clark, Lesgold, \& Winzenz, 1969; Cofer, 1965), but it is of some interest that the input effect was clearly present even though all conditions were provided with the category names at recall. The argument that blocked input facilitates recall simply by making it easier to identify categories (Cofer, 1965) is therefore not plausible. A more acceptable interpretation is that blocked presentation facilitates the encoding process. The facilitation of encoding might be expected from either structural or unitization models because the blocked input is more congruent with the structure of semantic memory. This congruence would make it easier to form appropriate units or rehearsal sets (Rundus, 1971), and consequently increase recall performance.

Of greater importance to our present concern is the finding that a good retrieval pattern (blocked) improved recall, while a relatively poor pattern (distributed) produced a decrement in recall. This result indicates that the test conditions are indeed important to recall and is consistent with the hypothesis that subjects develop a strategy of retrieval. If they find it difficult to develop and use an efficient retrieval system, as in the distributed condition, recall is depressed.

\section{EXPERIMENT II}

The fact that output order influences recall is quite consistent with the interpretation that subjects develop and use a retrieval strategy, but the effect might also be

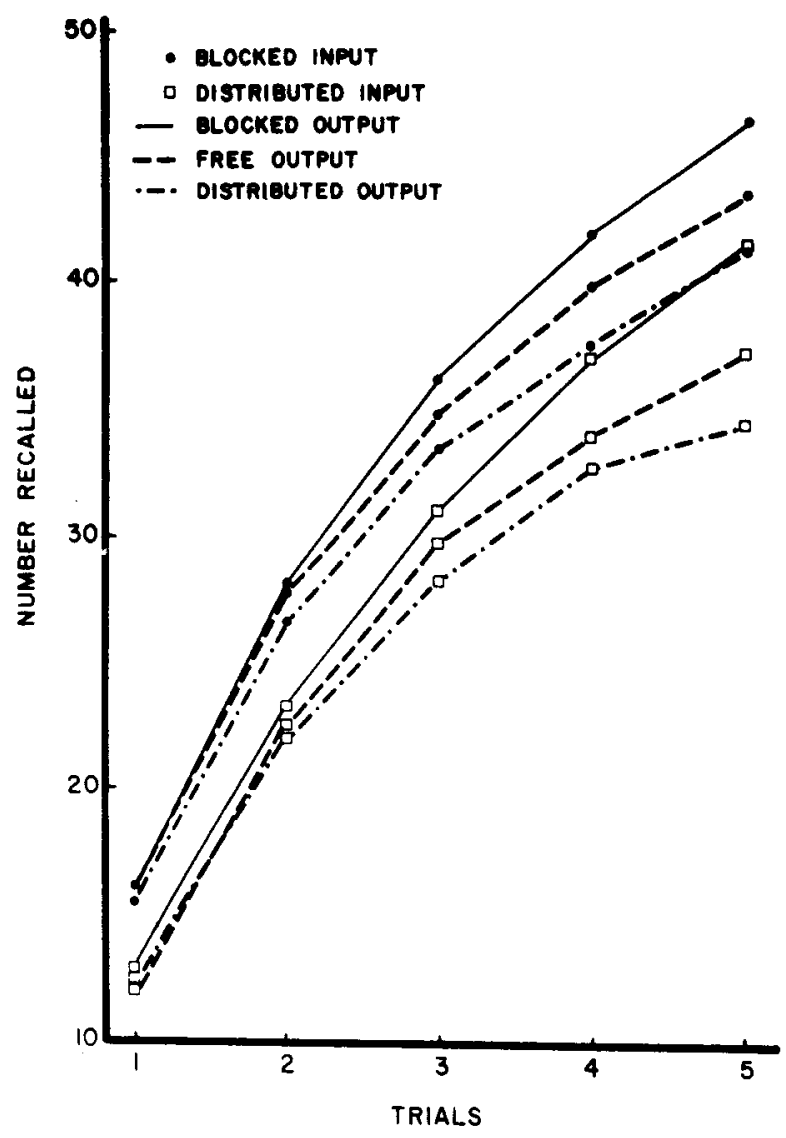

Figure 1. Mean recall as a function of input and output condition.

explained in other ways. An alternative hypothesis is that the recall test simply provides another opportunity to study the material. As the subject recalls the material, it enters the "spotlight of consciousness" and is in effect restudied (Anderson, 1972; Shiffrin, 1970). Therefore, requiring blocked output should lead to the same advantage as blocked input, permitting subjects to encode the test material more effectively.

One argument against this hypothesis is that manipulation of input and output pattern do not have identical effects in the first experiment. Blocking the input leads to an immediate and constant advantage which begins on Trial 1; whereas the advantage of blocked output is nonexistent on Trial 1, but grows gradually until it equals the input effect by Trial 5 . These data imply that the effects of output order are different from study effects and are consistent with the idea of a retrieval strategy being developed over trials. However, this argument against the test-as-study interpretation is rather weak in the absence of a reliable three factor interaction of Input by Output by Trials.

The second experiment was designed to evaluate the test-as-study-trial explanation. If a blocked test trial simply provides a better opportunity to study the list, then it should have no influence on current recall, but 
Table 1

Mean Recall as a Function of Blocked vs. Distributed Output Trials and the Order DB vs. BD

\begin{tabular}{lcccc}
\hline & \multicolumn{4}{c}{ Trials } \\
& 1 & 2 & 3 & 4 \\
\hline Order DB & & & & \\
$\quad$ Distributed Output & 20.28 & 31.84 & 37.22 & 40.53 \\
$\quad$ Blocked Output & 22.69 & 33.53 & 41.25 & 45.03 \\
Order BD & & & & \\
$\quad$ Distributed Output & 21.72 & 30.88 & 36.50 & 38.00 \\
$\quad$ Blocked Output & 24.41 & 35.15 & 41.88 & 45.34 \\
\hline
\end{tabular}

should facilitate subsequent test trials. In contrast, the retrieval strategy hypothesis asserts that the order of output should influence performance on the current test. Use of a good retrieval strategy should have an immediate influence on performance and should not be restricted to an effect on subsequent trials. The second experiment eliminates the potential test-as-study advantage of the blocked output condition by providing all subjects with both blocked and distributed test trials following each study period. Counterbalancing the test order (blocked or distributed first) across subjects eliminates the study advantage of blocked output because subjects on the averages received the same number of blocked and distributed tests. Therefore, if blocked output still leads to better recall than distributed, it implicates the importance of the retrieval process per se.

\section{Method}

Design and Materials. Each of four study trials was followed by both a blocked and distributed test trial. Thirty-two subjects received a blocked test trial followed by a distributed test trial for all four trials (Order BD) and 32 subjects had a distributed test trial followed by a blocked test trial for all four trials (Order $\mathrm{DB}$ ). The design was a 2 by 2 by 4 factorial of test order (BD vs. DB); output constraint (blocked vs. distributed); and trials. The word list was the same as that used in Experiment I. Study trials for all conditions were blocked with the order of the four categories counterbalanced across subjects and trials as in Experiment I.

The first category cued on blocked test trials was either the second or third category of the preceding study trial so that subjects never recalled words from a category they had just studied. This prevented any advantage accruing to blocked recall from a recency effect. For the distributed test trials, subjects always received the four category cues in the same order so they knew what category would be cued next.

Procedure. The subjects were instructed on the nature of the cued recall and tested individually. All subjects were given an initial presentation of the 60 -item list on a memory drum at a 2 -sec rate to familiarize them with the structure of the list. Following the initial presentation, four study-test trials wete given. Each trial consisted of presentation of the list by a tape recorder at a 3-sec rate followed by two test periods. The subjects were told which type of test trial they would receive immediately before the cueing sequence began. Recall was cued by lighting one of four indicator lamps located directly above the four category labels on a display $3 \mathrm{ft}$ in front of the subjects. Subjects were given both blocked and distributed tests following each study trial. The sequence of category cues was controlled by a Lafayette data system which presented the next cue light as soon as the subject recalled a word or after $5 \mathrm{sec}$ if no response was given.

\section{Results and Discussion}

The data are summarized in Table 1 as a function of blocked vs. distributed output trials, and the Order DB vs. BD. As in Experiment I, the main effects of trial and blocked vs. distributed output are highly significant, ps $<.001$, indicating that performance improves over trials, and that blocked output led to better performance than distributed output. The order in which tests were administered, BD vs. DB, had no influence on performance, $F(1,62)<1.0$. Order did lead to small but significant interactions with both output constraint, $F(1,62)=7.20, p<.01$, and trials, $F(3,186)=3.06$, $\mathrm{p}<.05$, but the interaction of Trial by Test Order by Output constraint was not reliable, $\mathrm{p}>.20$. In general, the interactions with the test order are uninteresting. The interaction of Order by Output Constraint arises simply because subjects do slightly better on the first of the two test output cycles. The interaction of Order by Trials is a small effect which is not easily or meaningfully interpreted.

Of primary concern in Experiment II is the interaction of Output Constraint by Trial. The data provide a highly significant $F(3,186)=14.12, p<.001$, replication of the interaction observed in Experiment $I$. Subjects perform better when they are allowed to recall in a good (blocked) pattern than when they are forced to recall in a pattern that interferes with the "natural" organization of words by category membership (distributed). Furthermore, the facilitation of blocked relative to distributed output increases over trials, resulting in a difference of more than $15 \%$ in recall by Trial 4.

The most important aspect of these data is that the output effects are obtained in a design that is not consistent with the hypothesis that blocked output merely produces a better means of restudying the list. The prior history of study and test trials was completely equated across output conditions. Therefore, the experiment indicates that encoding and study effects are not sufficient to account for the recall advantage of blocked output. Subjects seem to be learning a retrieval strategy which can influence performance indpendently of input effects. This retrieval strategy appears to develop over trials and can be used on the blocked test trials but is not useful on the distributed tests.

\section{EXPERIMENT III}

The first two experiments clearly demonstrate the importance of a retrieval pattern in recall. Memory is not strictly determined by the conditions of encoding, but is also influenced by the circumstances of retrieval. Subjects remember more on a given trial when they are allowed to proceed in a conceptually ordered fashion 
than when forced to recall across semantic categories. This result implies that subjects do not simply encode, rehearse, and strengthen individual items, but also develop a method or plan of retrieval. These data require structural models to acknowledge the importance of the retrieval process. However, it might be possible to explain the effect of output pattern with a relatively simple elaboration of a structural model such as Anderson's FRAN. FRAN has an associative memory which contains some degree of conceptual organization because items within categories tend to be closely associated. Therefore, it is possible that the distributed output interferes with recall by forcing subjects to monitor simultaneously (or keep track of) the examined (or marked) pathways and words from several categories. Under such circumstances, subjects are likely to "lose their place" and have to search previously examined pathways to obtain a new word. In the blocked condition, the subject remains within a restricted memory region. In other words, distributed output creates a bookkeeping problem, forcing subjects to remember which pathways and words have been examined in all categories.

The bookkeeping explanation allows a structural model to account for the effect of output pattern without invoking the concept of a retrieval strategy. The problem, then, is to differentiate such structural models which are sensitive to output order from strategy models which do not rely on the permanent structure of a semantic memory. The basic approach is to test the ability of a structural model to account for strategy effects in a situation where bookkeeping problems are eliminated. The test can be accomplished with a modification of a task first reported by Bower, Clark, Lesgold, and Winzenz (1969). They presented one group of subjects with word sets arranged spatially in natural conceptual hierarchies. Another group of subjects received the same word sets placed randomly in the spatial positions of the hierarchy. The group shown the conceptual structure recalled approximately three times as many words as the control condition on the first recall trial. The Bower et al. (1969) experiment can be modified so that subjects first recall as much as possible and are then given words from the hierarchy to cue further recall. These cues can be given so they reveal the conceptual structure, or in a way that simply gives subjects the identical set of words as cues, but fails to specify the list structure. The interesting comparison in such an experiment is between conditions given randomly arranged input followed by cues which reveal, or fail to reveal, the conceptual structure.

The strategy interpretation of the retrieval process suggests that providing subjects with a good method of recall, even during the test should improve performance. Information about list structure could be used to alter the retrieval strategy and generate recall more efficiently. On the other hand, an associative net model, even with the bookeeping provisio, predicts that giving subjects entry to the cue words in the structural network should produce more recall, but the structural information should be irrelevant. According to a structural model such as FRAN, retrieval cues are effective because they provide access points to the semantic mernory. However, providing information about list structure should have no additional effect because all of the structural information is contained in the semantic network. If the same cue words are presented, they provide access to the same areas of the associative network, irrespective of the presentation form. Thus, both models predict a positive effect of cues, but only the retrieval stragegy hypothesis suggests that the hierarchical conceptual structure presented with the cues will increase performance. Providing both groups with the same cues eliminates bookkeeping problems because the network activated from the locus of each cue word. Structural information is contained in the network, rather than as a retrieval strategy.

Experiment III employs this postrecall cueing technique to examine the role of strategy in retrieval.

\section{Method}

Materials. The 124 stimulus words consisted of four conceptual hierarchies used by Bower et al. (1969) (parts of the body, modes of transportation, types of plants, and types of animals). The stimulus words were shown in four sets of about 30 words each presented either in hierarchical or random fashion. In hierarchical form, each of the four stimulus arrays was a spatially organized natural hierarchy of four levels, as in the Bower et al. (1969) experiment. In the random presentation, each of the four stimulus arrays preserved the spatial layout of a hierarchy, but the words were randomly chosen to fill the positions from the entire set of 124 words.

Design. There were three basic conditions in the experiment determined by the type of stimulus presentation, and type of postrecall cue. The subjects in the hierarchy-hierarchy condition were presented with the four word sets, each in hierarchical form. They were then told to recall, in any order, all of the words they could remember. After this initial recail attempt, subjects were shown the top three levels of each hierarchy, and were asked to add to their recall as many previously unrecalled words as possible.

The two remaining groups were presented with the same word sets in random fashion. Subjects in the random-hierarchy (RH) condition were allowed to recall all of the words they could from the four displays and were then cued with the top three levels of each conceptual hierarchy. They were told to add as many previously unrecalled words as possible with the aid of the hierarchy cues. Subjects in random-random (RR) were also presented with the four arrays in random fashion, asked to recall as many words as possible, and then cued with the same set of cue words as the other two conditions. However, in Condition RR, the 39 cue words which comprised the top three levels of the four conceptual hierarchies were randomly assigned to three lists of 10 and one list of 9 cue words. Thus, in both the random and hierarchy cueing conditions the same set of words served as postrecall cues. The only difference was that the hierarchical structure was shown in Conditions $\mathrm{HH}$ and $\mathrm{RH}$, but no structural information was given to Condition RR.

A total of 36 undergraduates were paid to participate in the experiment. Subjects were tested in small groups of five to seven with two groups assigned to each treatment condition. 
Table 2

Mean Recall as a Function of Presentation Cue Format

\begin{tabular}{lccc}
\hline Condition & $\begin{array}{c}\text { Original } \\
\text { Recall }\end{array}$ & $\begin{array}{c}\text { Words } \\
\text { Added }\end{array}$ & $\begin{array}{l}\text { Intru- } \\
\text { sions }\end{array}$ \\
\hline Random-Random & 31.69 & 4.85 & 4.18 \\
Random-Hierarchy & 31.23 & 10.23 & 4.92 \\
Hierarchy-Hierarchy & 42.35 & 19.00 & 2.10 \\
\hline
\end{tabular}

Procedure. Subjects in each condition were shown four successive arrays of words belonging to the four conceptual hierarchies. In Condition $\mathrm{HH}$, subjects were told that the words would appear in the form of four spatially arranged conceptual hierarchies. The subjects in Conditions RH and RR were told that the words would appear spatially spread out on the screen, and were cautioned that the placement of the words was arbitrary. All subjects were told that the spatial location of the words was irrelevant and, that they would not be required to remember where a word had occurred.

The four stimulus arrays were presented on a screen with an overhead projector and were shown successively, each for $1 \mathrm{~min}$. At the end of the last presentation, subjects turned to their problem booklets and solved long division problems for $90 \mathrm{sec}$. Subjects then recalled by writing, in any order, all of the words they could remember from the four arrays. They were allowed $7 \mathrm{~min}$ to recall, which was sufficient for all subjects. At the end of the recall period, subjects were given cue words to prompt further recall. In condition $\mathrm{RH}$, subjects were told that the words they had been shown were in fact drawn from four conceptual hierarchies. The subjects in Condition $\mathrm{HH}$ and $\mathrm{RH}$ were then presented with the top three levels of each conceptual hierarchy, and were told to use this conceptual structure to help them think of any Level 4 instances not previously remembered. In Condition RR, the same cue words were used except they were randomly assigned to four lists, and presented without an indication of spatial or conceptual structure.

Each of the four sets of cue words was presented for $90 \mathrm{sec}$, and subjects then wrote any additional words recalled during the 6-min cued recall.

\section{Results and Discussion}

Table 2 shows the mean number of words recalled during both the initial and cued recall periods. Extralist intrusions which occurred during cued recall are also shown. An unweighted means analysis of variance indicates that presentation in hierarchical form facilitated recall, $F(2,33)=3.35, p<.05$, replicating the results of Bower et al. (1969). In their experiment, however, the effect of hierarchical presentation was considerably more dramatic.

Of primary interest are the differences among conditions during the cued recall period. An analysis of variance of words added during the cue period indicates that there were reliable differences among the means $F(2,33)=17.87, p<.01$. The contrasts of RR vs. RH, $F(1,33)=5.90, p<.05$, and $\mathrm{RH}$ vs. $\mathrm{HH}$, $\mathrm{F}(1,33)=13.66, \mathrm{p}<.01$, were both significant. All of the conditions were able to add words to their recall, and there were no reliable differences in number of intrusions, $F(2,33)<1.00$. Also, it might be pointed out that a simple correction for guessing (hits minus intrusions) suggests that there is no real improvement in Condition RR. This ordering of test cue effectiveness is not predicted by a structural model because all conditions were given equal access to the cue words. Therefore, the structural information does not seem to be contained in a permanent network representation which is marked for recall. The increase in effectiveness with structural information suggests that subjects are fully capable of employing a good retrieval strategy, even when provided only in the test phase.

\section{GENERAL DISCUSSION}

The experiments have all shown a clear influence of test circumstances on recall. They demonstrate a general effect of retrieval cues and, in particular, show that the structure and organization among cues is important to recall.

In addition to documenting the importance of retrieval effects in learning, the data also have bearing on general models of the memorial process. The introduction briefly discussed two classes of recent memory models: structural and process oriented. First, consider the structural position embodied in Anderson's FRAN, which ascribes learning to the "tagging" of nodes and pathways in an existing semantic network. This type of model has considerable difficulty with a variety of test trial effects, for example: (a) Slamecka (1968) found that providing a subset of the recall list as retrieval cues had no effect on subsequent recall. Providing FRAN with such cues led to large increases in recall. (b) Hogan and Kintsch (1971) found that test trials led to better delayed recall performance than did study trials. (c) In the present experiments, the form of retrieval had large effects on recall, and providing subjects with a hierarchical retrieval strategy led to better performance than simply giving subjects access to the same cue words.

The present data also are inconsistent with an independent trace notion of memory put forth by Slamecka (1968). The independent trace hypothesis suggests that items exist in memory with no particular connectivity or oder. Recall proceeds by selecting an appropriate retrieval strategy to generate potential items. This type of model based strictly on a retrieval strategy also has difficulty with the results of Experiment III. The model predicts that subjects should gain by having a good plan of recall, and this effect was observed in Experiment III. Both conditions, $\mathrm{RH}$ and $\mathrm{HH}$, recalled more words in the presence of structural recall cues. However, Slamecka's retrieval model also predicts that both Conditions $\mathrm{RH}$ and $\mathrm{HH}$ should gain roughly the same amount since they were given identical retrieval strategies at recall. However, when the structural cues were presented both at study and test (HH) they were nearly twice as effective; a result incompatible with the concept of independent traces.

The only model which remains viable is a derivative of the unitization model which captures the notion of a 
retrieval strategy and yet does not deny the existence of a permanent structured knowledge base. A reasonable version of the model would employ a working memory similar to Tulving's episodic memory. The working memory provides a context-specific area in which subject attempts to establish a set of rules, algorithms, and connections for remembering the current material. Such contextual memories are not isolated from the permanent knowledge structure, but are an integral part of it, providing the factual basis of semantic memory.

The easiest way to explicate the model is to consider an individual faced with the task of learning an arbitrary list of words. First he encodes the distal stimulus into an internal representation consistent with his semantic knowledge of the word. The subject extracts certain features of the permanent representation, and establishes a code for the word in his working memory. The important point is that the working code is impoverished, without all aspects of the permanent representation and the rich interconnections with other concepts of the semantic memory.

As the subject forms a working code, he in fact establishes the minimal retrieval plan by creating a temporal sequence of representations that might be used to guide recall. Such a temporal sequence is the lowest level of recall strategy and is most likely involved only under the ecologically bizarre demands of the laboratory situation. A temporal relationship ordinarily exists only because the subject holds material in an effort to abstract more efficient methods of access. While holding the temporary codes, the subject usually establishes connections among items based upon semantic associations and relationships. A common example is the attempt to combine associatively related items, or form images that integrate sets of items into meaningful scenes. A higher level strategy involves an attempt to extract features or groupings that characterize the list, as evidenced by categorical or hierarchical organization of recall. Finally, if possible, an attempt is made to eliminate all particulars from the working memory by creating a set of generative rules. This last situation often obtains in problem solving and concept-identification paradigms. For example, in a concept task, subjects typically use information about prior instances to formulate a rule for partitioning the stimulus population, but once the rule is formed the particular examples are no longer retained. Subjects seem to respond strictly on the basis of the rule without reference to the prior history of exemplars.

This type of strategy model emphasizes a heavy interaction between the working memory and the semantic knowledge base. The model differs from structural models in that it creates a certain measure of independence from the semantic memory, and emphasizes a flexible generative system. It differs from Tulving's episodic memory by allowing a great deal of interaction with semantic systems beyond the initial encoding process. Tulving stresses the creation of an episodic memory code tied to particular retrieval cues. The current model suggests a working memory that contains a retrieval scheme rather than simply a collection of specifically encoded traces. The model is admittedly vague on the specific nature of the retrieval plan. A good deal more experimental work will be required to explicate fully the potential rules of retrieval. However, an intuitive example in the context of the present experiments would be that the subject attempts to establish units or subgroupings of the list items. The retrieval strategy might then consist of a set of instructions specifying the nature of the entire list. This form of strategy model where subjects store generation instructions and list characteristics is useful to explain a wide variety of recall effects. For example, the important of test trials in recall tasks (Hogan \& Kintsch, 1971) can be ascribed to the test which provides feedback on the effectiveness of the retrieval strategy. Similarly, in the present experiments the strategy model provides a reasonable explanation of the effect of output pattern. A distributed test trial makes it much more difficult to form units in the temporary memory, and create instructions to retrieve the subgroups. Such a model also predicts the results of the third experiment in which a good retrieval strategy was effective when provided only during recall (Condition $\mathrm{RH})$.

The strategy model basically suggests that cues should be most effective if they provide a sensible way to access the various parts of the memory list. It is, therefore, reasonable that category names (Tulving \& Pearlstone, 1966; Wood, 1967) or the top levels of a conceptual hierarchy (Experiment III) are particularly effective recall cues. One would also expect much less effect of cues if they do not contain information about list structure, e.g., Slamecka (1968). In fact, Slamecka's procedure of cueing with a random subset of the list might be more disruptive than useful because the cues do not contain a recall strategy and they prevent subjects from using whatever strategy they had originally developed. The situation is similar to the present experiments in which subjects who were given no recall cues (free output) did better than those forced to use Distributed cues.

Finally, the unitization-strategy model provides a reasonable explanation of the recent data on encoding specificity (Santa \& Lamwers, 1974; Thomson \& Tulving, 1970; Tulving \& Osler, 1968; Tulving \& Thomson, 1971, 1973). Tulving and his associates have suggested that subjects encode items into an episodic memory with specific cues for retrieval. They demonstrated that such memories cannot be accessed by strong associate cues unless the cues were encoded with the items during study. However, Santa and Lamwers (1974) have shown that subjects can make use of such associates if they are simply provided with a strategy for their use. Subjects' performance is greatly improved 
when they are told to generate high associates of the cue words. The same cues are completely ineffectual without instructions. The best interpretation of these experiments is that subjects encode items into a working memory, and they can flexibly access this material if given a good retrieval strategy.

In summary, the strategy model outlined in the present paper has large advantages over both structural and simple retrieval models. The model is particularly useful to explain the variety of effects obtained in cued recall paradigms.

\section{REFERENCES}

Anderson, John R. FRAN: A simulation model of free recall. In G. Bower (Ed.). The psychology of learning and motivation, Vol. 5. New York: Academic Press, 1972.

Battig, W. F., \& Montague, W. E. Category norms for verbal items in 56 categories: $A$ replication and extension of the Connecticut Category Norms. Journal of Experimental Psychology Monograph, 1969, 80, 1-46.

Bower, G. H., Clark, M. C., Lesgold, A. M., \& Winzenz, D. Hierarchical retrieval schemes in recall of categorized word lists. Journal of Verbal Learning and Verbal Behavior, 1969 , 8. 323-343.
Hogan, R. M., \& Kintsch, W. Differential effects of study and test trials on long-term recognition recall. Journal of Verbal Learning and Verbal Behavior, 1971, 10,562-567.

Santa, J. L., \& Lamwers, L. L. Encoding specificity: Fact or antifact. Journal of Verbal Learning and Verbal Behavior, $1974,13,412-423$.

Shiffrin, R. M. Memory Search, in D. A. Norman (Ed.), Models of human memory. New York: Academic Press, 1970.

Slamecka, N. J. An examination of trace storage in free recall Journal of Experimental Psychology, 1968, 76, 504-513.

Thomson, D. M., \& Tulving, E. Associative encoding and retrieval: Weak and strong cues. Journal of Experimental Psychology, 1970, 86, 255-262.

Tulving, E. Episodic and semantic memory. In E. Tulving \& W. Donaldson (Eds.), Organization of memory. New York: Academic Press, 1972.

Tulving, E., \& Osler, S. Effectiveness of retrieval cues in memory for words. Journal of Experimental Psychology, 1968, 77, 593-601.

Tulving, E., \& Pearlstone, Z. Availability versus accessibility of information in memory for words. Journal of Verbal Learning and Verbal Behavior, 1966, 5, 381-391.

Tulving, E., \& Thomson, D. M. Retrieval processes in recognition memory: Effects of associative context. Journal of Experimental Psy chology, 1971, 87, 116-124.

Tulving, E., \& Thomson, D. M. Encoding specificity and retrieval processes in episodic memory. Psychological Review, 1973, 80, 352-373.

Wood, G. Category names as cues for the recall of category instances. Psychonomic Science, 1967, 9, 323-324.

(Received for publication August 16, 1974; revision accepted September 19,1974.) 